\title{
Knowledge-based design environment for primary shaped micro parts
}

\author{
A. Albers, N. Burkardt, S. Hauser, J. Marz
}

\begin{abstract}
This contribution describes a prototypical knowledge-based design environment predominantly concentrating on the production-relevant design of micro parts. In this environment a computer-aided knowledge base is linked with a design system (CAD-system). In contrast to a complete knowledge-based design, i. e. modelling a part by the rule-based connection of distinct basic objects or features (which is a matter of structured combination rather than creative designing) the prototype presented promzotes conventional practical design. The designed part is subsequently checked on the basis of parameters derived from the geometry of said part.
\end{abstract}

Keywords Knowledge-based engineering, design environment, design rules, elementary rules

\section{1}

\section{Introduction}

Today, production technologies can be regarded as the focus of the product development process of micro parts. The process-specific parameter and requirements define the smallness as well as the obtainable qualities of parts. Due to the progressing development of micro technology and the increasing market penetration by medium and large batch products in product development also process steps previous to or following the production become more and more relevant to an effective design of the machine element, which is to meet the respective requirements. As a result, supporting the designer by means of basic technological knowledge and experience, which is not limited to individual people, becomes necessary.

The work presented in the following demonstrates the state of development of a knowledge-based design environment adapted to the specific requirements of hea-

Received: 8 August 2003 /Accepted: 6 November 2003

A. Albers, N. Burkardt, S. Hauser, J. Marz ( $\square)$ Institute of Machine Design and Automotive Engineering, University of Karlsruhe, Kaiserstrasse 12, 76128 Karlsruhe, Germany e-mail: marz@mkl.uni-karlsruhe.de

This paper was presented at the Fifth International Workshop on High Aspect Ratio Microstructure Technology HARMST 2003 in June 2003. The work is part of the DFG (Deutsche

Forschungsgemeinschaft) funded collaborative research center 499 "Development, production and quality assurance of primary shaped micro components from metallic and ceramic materials" vy-duty micro parts molded from metallic and ceramic materials. The overall aim is to interpret the results and information achieved in the collaborate research center 499 from different disciplines relevant to design and ensure their computer-aided availability. In doing so, the CAD system, which is familiar to the designer, is to support him during the design process.

\section{2}

\section{Knowledge-based design}

Up to now, several commercial systems for knowledgebased design support have been available on the market [9]. In these systems the rules refer to exactly defined dimensions of the final design and are thus mainly limited to the support of modifications. Furthermore, the rules can generally not be applied to other parts as they are exclusively integrated into the data record of the respective component.

\section{3}

\section{Rule-based design methodology for micro parts}

A knowledge-based system is constituted by a knowledge base. It contains factual knowledge about production technologies as well as the rules for connecting manufacturing properties and geometric parameter. Since both are independently subject to modifications they consequently need to be formulated separately. The manufacturing properties result directly from the technologies currently available and change thus in accordance with the respective state of the art of machine tools or production engineering. The rules are obtained due to the experience of the designer, the process preparation and the production. Therefore, it constantly undergoes further developments and supplementations. Apart from that both types of knowledge are to be filed independent of the CAD system. The resulting advantage is that the same information can be updated also without CAD and used for different CAD systems.

\section{1}

\section{Restrictions of production}

The currently applied rules exclusively refer to the production process chain of primary shaped micro parts. Hence, it must be considered that as the model is mostly created in the CAD, a negative mold - the mold insert is automatically generated. With parametric CAD systems it is possible to consider the sinter shrinkage in this process and to correspondingly create a bigger mold insert. The production of the model is then carried out in two steps. 
Firstly, the mold insert (negative) is manufactured. The technologies currently available for this process are micro milling and micro erosion [8]. The second step consists of molding the model (positive) by means of ceramic or metallic micro powder injection molding or micro casting $[6,7]$.

On both process steps there are separately different restrictions, e.g. that it is not possible to mill a cavity, which is smaller than the milling cutter or that in terms of the micro powder injection molding a minimum wall thickness and a maximum flowing distance need to be observed. However, both parts are obviously geometrically connected, i.e. restrictions on mold insert production automatically also concern the molded part and vice versa. In this context it is vital to consider that the geometric properties of the two parts are not identical. Firstly, the part is scaled during the molding process due to the sinter shrinkage and secondly, complementary structures are developed because of which e.g. a bore in the mold insert transmutes into a cylinder in the model with smaller height and diameter.

\section{2}

\section{Presentation of the rules}

\subsection{1}

\section{Classification}

The knowledge-based design environment makes it possible to separately formulate the rules for different process steps. However, it is necessary to unambiguously point out to which type of machine element the geometric quantities relate. This can be achieved by means of a so-called rule class, which indicates for which type of machine element a rule has been set up and to which production technologies and tools or materials it applies.
If a rule is applicable to all technologies or tools/materials the marking " $x x x$ " or " $x$ " can be observed instead of the special code. Due to an additional consecutive number an unambiguous rule code for each rule is derived (Fig. 1).

\subsection{2}

\section{Technology-related design rules}

In order to point out the relation to production technologies the design rule KR_FE_MF3_x_002 is explained as an example. It refers to the production technology of 3-axle micro milling preparing the process, which applies to various tool groups (end mill cutter and radius form cutter). It relates to the mold insert and is the second of this rule class combination. The corresponding mathematical expression is as follows:

$R_{\text {inner_edge }} \geq \frac{d_{\text {milling_cutter }}}{2}+T_{\text {mill }}$

Because of the circular cross section of the milling cutter no vertical inner edges or radii, which are smaller than the milling cutter's radius plus the milling tolerance, can be produced. However, if the radius is as big or even bigger than the milling cutter, the tool can process to the geometry of the cavity. Applied to the geometry of a micro gear (Fig. 2) this implies a tooth tip rounding and thus a reduction of the effective transverse contact ratio. $[1,4]$

\section{4}

\section{Approach for description and routines for evaluation}

\section{1}

\section{Geometric approach of boundary representations}

In accordance with the aim of the collaborative research center (SFB 499) to produce micro parts in midium to large scale, this application is to examine whether the

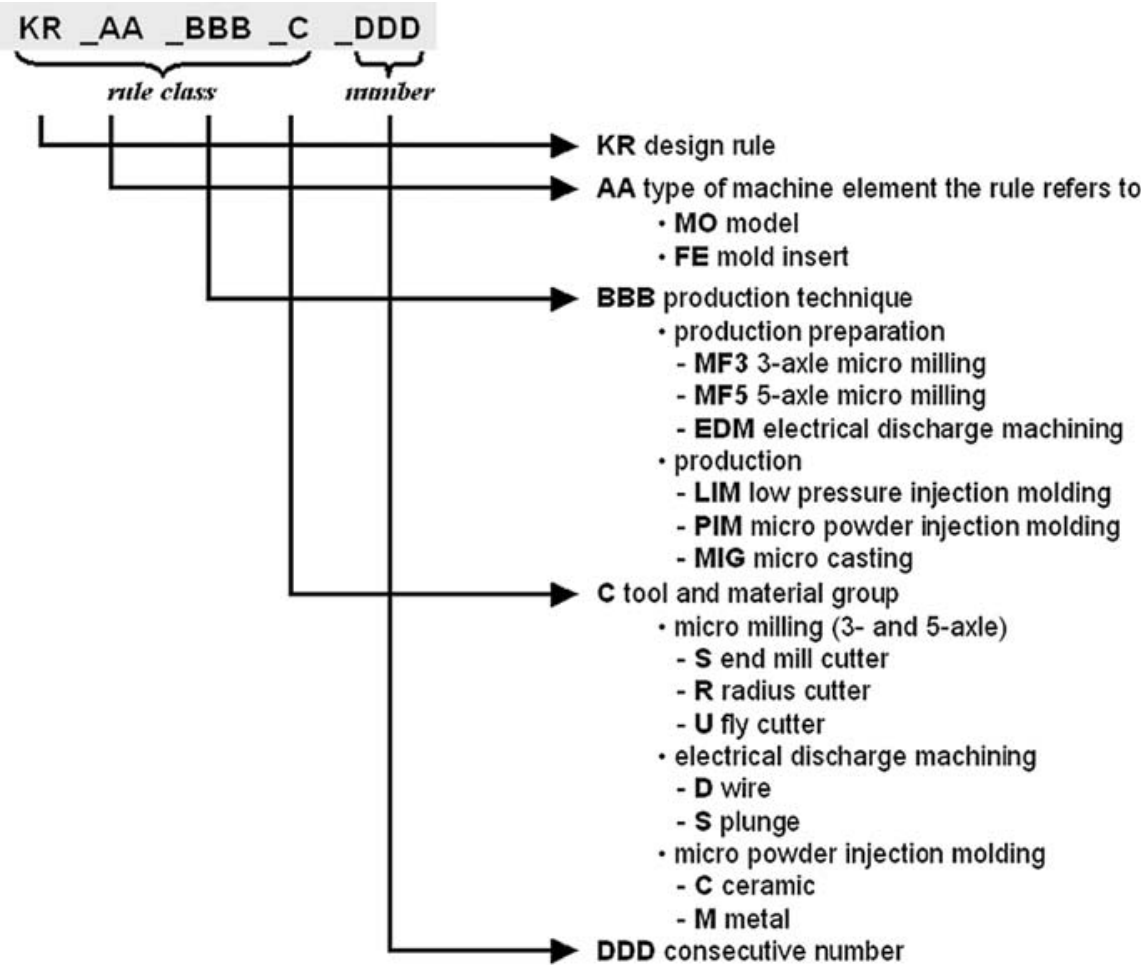

Fig. 1. Unambiguous rule code formed from a rule class and a consecutive number 


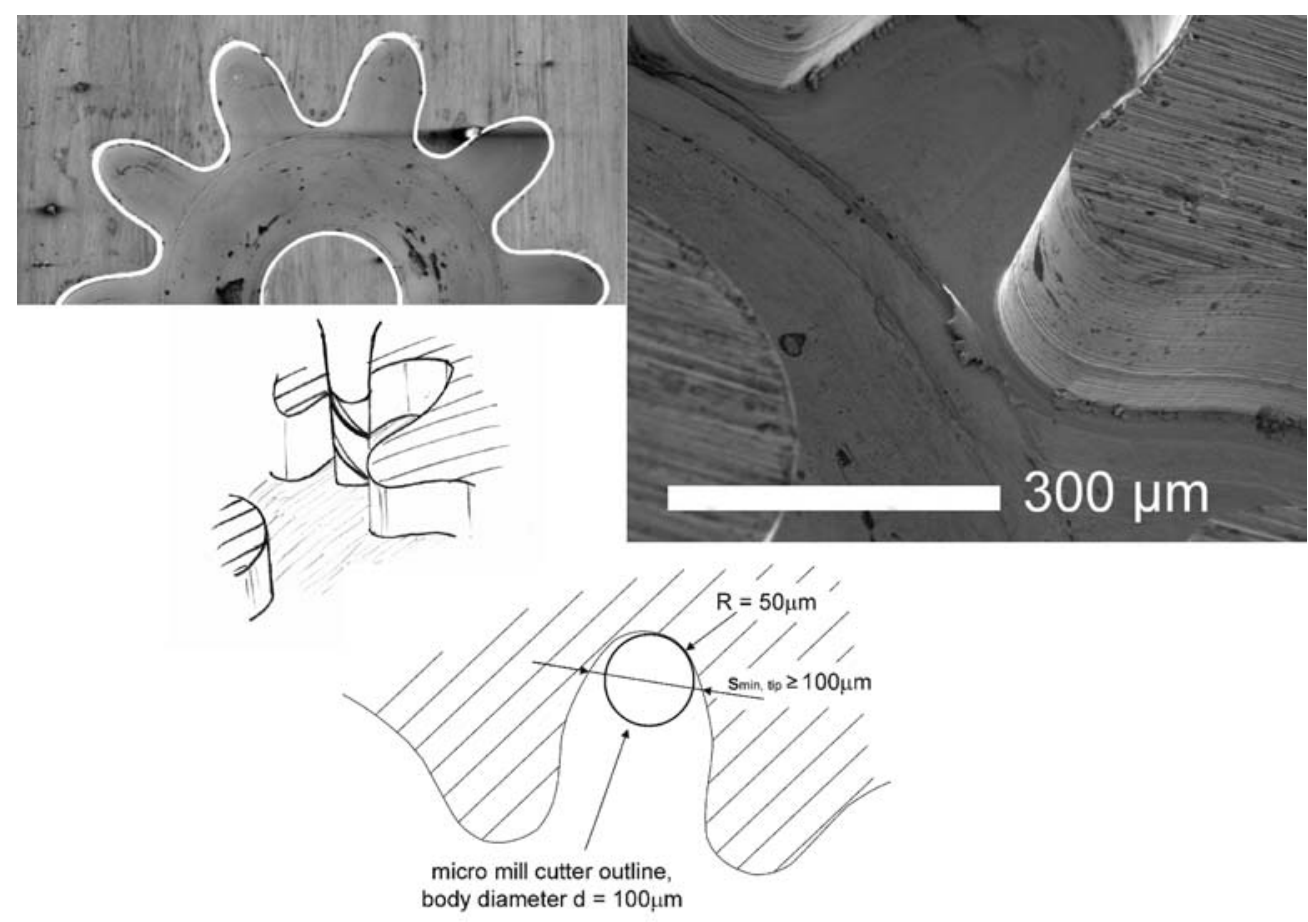

Fig. 2. Rounding of the inner edges of a mold insert by the used micro-milling

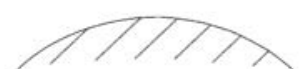
cutter due to manufacturing

designed geometry can be manufactured by means of the production technique and tool selected. Therefore, the necessity to focus only on geometry is obvious. Strictly speaking, in the production process not volume-oriented features, such as a cuboid or a cylinder, but individual surfaces and edges are generated. As a result, a description by means of boundary representation (B-rep) [2] has been chosen as the geometric model. In addition to that, many common transfer formats for CAD data such as STEP [3] or IGES support this description model.

The task of a programmed algorithm is thus to derive geometric parameter from the three-dimensional geometric model and connect them by means of rules with the production technological parameter.

\section{2}

\section{Adapting and evaluating rules}

By adapting general rules to the special geometry as well as to the individual production process elementary rules are obtained. Consequently, they are not generally applicable anymore, but describe concrete circumstances and can hence be evaluated by the computer. As can be seen in Fig. 3, the preparation for this is carried out in two parallel steps: the adaptation and concretization of the rules and the determination of the geometric parameter.

The processing of the rules as provided by a database until the time, when they can be connected with the geometric parameter, is carried out in three steps.

1. The rules are loaded from a database into a list corresponding to their rule code. Rules, which are not appropriate according to the technology or the tool/ material are not considered.
2. If a rule is defined for the other type of machine element (model <->mold insert), it is "translated" by means of a transformation table. In so doing, certain key words are replaced by others (e.g. inner diameter $>$ outer diameter)

3. In accordance with the applied production process chain the production technological data are loaded from a separate database after which the respective placeholders in the rules are replaced. If for a placeholder no equivalent can be found in the database, the value " 0 " is assumed.

\subsection{1}

\section{Evaluation of geometry}

The evaluation of the geometry of the machine element is carried out in two phases. Firstly, all boundary representations from the database of the CAD system need to be read and secondly the corresponding properties of each representation are to be determined.

Geometric properties can be defined for solids, individual surfaces, two surfaces or edges (Fig. 4). For the determination of a property geometric information about the individual boundary representations from the part database are available, such as unit (normal) vectors, fulcrum, boundary representations etc. From these simple geometric parameter can be derived directly. Two surfaces e.g. are parallel when they have identical unit normal vectors.

For other parameter the calculation is more complicated, since there are various influencing factors or interdependence. One example is the angle $\alpha$ of an edge, which is defined as the angle measured by the part between 


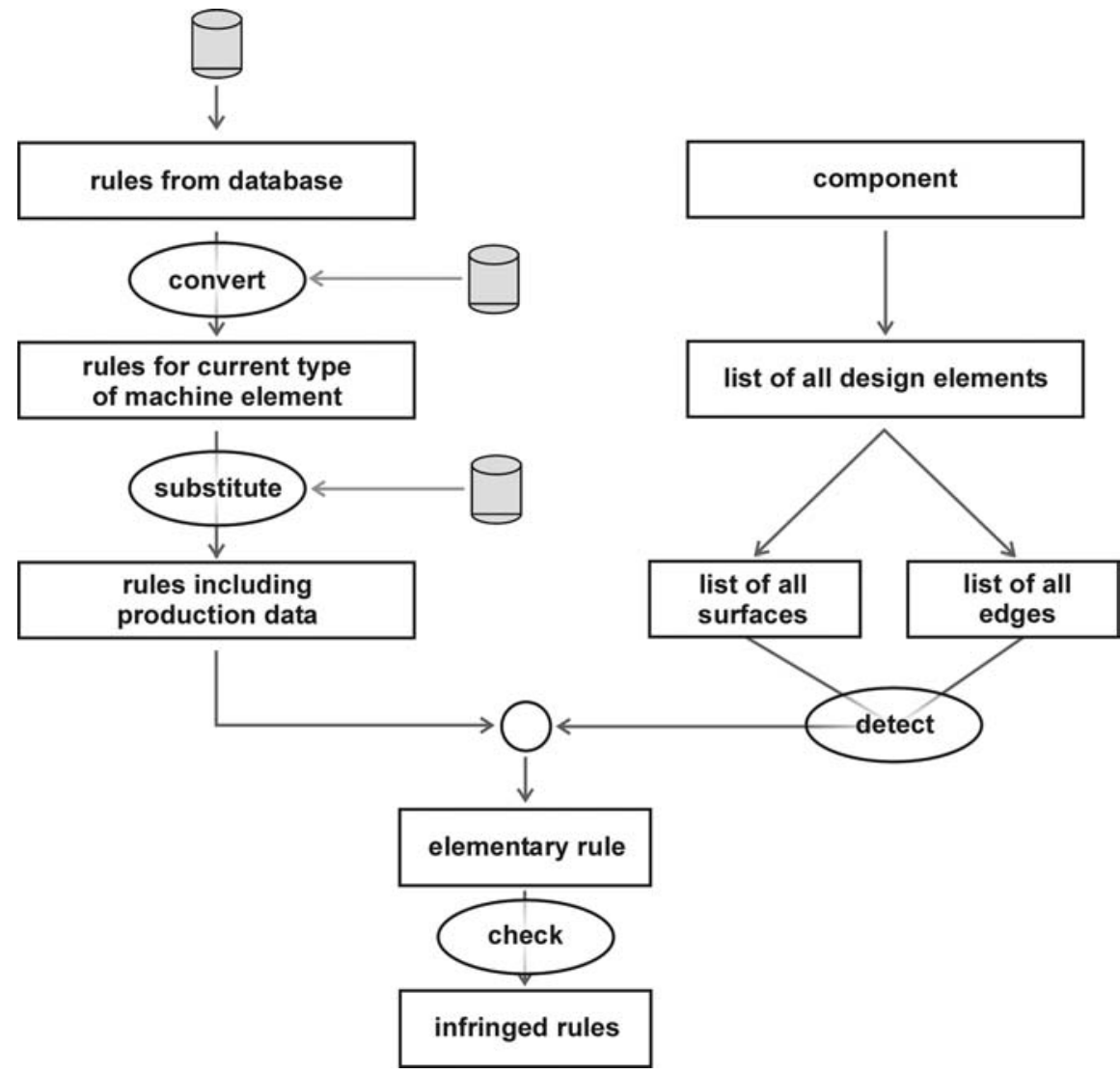

Fig. 3. Derivation of elementary rules from general design rules and the component's geometry

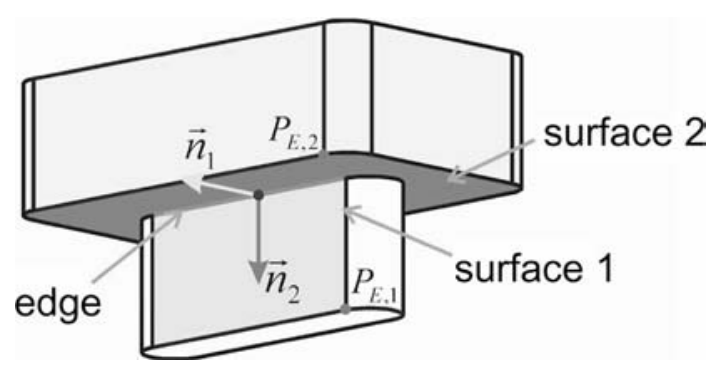

Fig. 4. Determination of the boundary representations and their relations

the adjacent surfaces directly at the edge. For the determination of this quantity the fact that normal vectors are always indicated outwards from the volume solid of the CAD is used.

In particular it is calculated in the following steps:

1. Determination of the adjacent surfaces

2. Specifying a common point of the surfaces on the edge

3. Determination of the unit normal vectors of two surfaces at this point

4. Calculation of the angle between the unit normal vectors $\cos \varphi=\vec{n}_{1} \cdot \vec{n}_{2}$

5. Determination of the small angle between the surfaces: $\alpha^{*}=180^{\circ}-\varphi$

6. Adjustment of non-parallel surfaces $\left(0^{\circ}<\alpha^{*}<180^{\circ}\right)$, if it is an inner edge $\left(\alpha>180^{\circ}\right)$ or an outer edge $\left(\alpha<180^{\circ}\right)$.
7. Check if one of the surfaces exceeds the common edge in the direction of the other normal vector. This is possible by determining the turning point $P_{E, 1}$ on the surface $F_{2}$ in the direction of the normal vector $\vec{n}_{2}$ of the other surface $F_{2}$. To obtain an obvious point two further directions need to be defined. For this the cross product $\vec{n}_{1} \times \vec{n}_{2}$ and the first normal vector $\vec{n}_{1}$ are used. The point $P_{E, 2}$ is determined analog.

8. Adjustment if one of these two points is on the common edge of the surfaces; if yes, then it is an outer edge, if not, it is an inner edge.

9. Calculation of the angle:

with an outer edge: $\alpha=\alpha^{*}$

with an inner edge: $\alpha=\alpha^{*}+180^{\circ}$

\subsection{2}

\section{Deriving and evaluating the elementary rules}

The detailed rules obtained by means of the steps listed above are now combined with all applicable boundary representations and their geometric properties in order to derive elementary rules. The latter only consist of numbers and mathematical operations and can be evaluated by an appropriate programme.

5

Commercial software modules as a basis for the knowledgebased design environment

\section{1}

\section{The 3D CAD system Unigraphics V18.0}

For realising the knowledge-based design environment the commercially available system components are to be used 
to a great extent. Unigraphics (UG) V18 of the company Unigraphics Solutions, Inc. [9] is employed as CAD system as it offers the possibility of a full parametric design. Furthermore, since version 17 a knowledge-based module "UG/Knowledge Fusion" (UG/KF) as well as a programmable user interface ("UG/Open API") is provided. The advantage of directly using a CAD system with an integrated knowledge-based module is that no neutral interface formats are required. As a result, costs and effort can be reduced and a loss of information (e.g. parametric or attached attributes) due to several format conversions can be averted.

\section{2}

\section{Knowledge database}

The filing of the rules and production technologies is realised with Microsoft Access. As a common database system its functionality is absolutely sufficient for the amounts of data, which is to be administered in this prototype. Moreover, it enables easy access to the data by means of the programme written in Microsoft Visual C++ 6.0 for the checking of the part.

\subsection{1}

\section{Production database}

The production technological parameter are stored in a separate database. Especially in the micro section they change constantly as there are always new techniques available or existing ones are improved. The filing is carried out separately after the production preparation, i.e. technologies for the generation of the mold insert, and the actual production process, the molding. In the production preparation not only the production technique but also the tools are distinguished. For each of those any properties can be defined, which are filed in a separate, connected table. Thus, new properties can be introduced via the database only by means of modifications.

\subsection{2}

\section{Rule database}

In a rule database all defined design rules are filed. They are contained in the form of an explanatory text, which is also displayed in case of a rule infringement, as well as in the form of an "IF ... THEN ... ELSE" condition. The "IF" part is formulated as a mathematical equation with placeholders for production technological and geometric parameter. If the described equation is correct, the "THEN" part is carried out, if not, the "ELSE" part, which may be e.g. an automatic correction [5].

\section{6}

\section{Knowledge-based design environment for micro parts}

\section{1}

\section{Programme flow}

Before it is possible to check the micro part the designer needs to indicate the used production technologies and the type of machine element of the product model, which is to be designed. For this purpose, in a pop-up window a question whether the mold insert or the model is involved and a question concerning the techniques and tools planned for the production are displayed. As a consequence, the corresponding rule classes can be derived. This information is saved together with the product model in the CAD file.

The checking is to a great extent carried out by means of a $\mathrm{C}++$ programme, which has been written exclusively for this task. In this programme the CAD data and databases are read in and elementary rules are created. These elementary rules are checked in the module UG/KF and rule infringements are presented in a pop-up window.

\section{2}

\section{Demonstration of functionality}

\subsection{1}

\section{Demonstration part}

In order to demonstrate functionality a micro mechanical part has been defined, with the aid of which many production restrictions can be presented. It is a short T-piece at which the web has been shortened a little bit and the vertical edges are rounded so that the mold insert can be milled. The mold insert is to be milled with an end mill cutter ( $\varnothing 200 \mu \mathrm{m}$, length of cutting edge $400 \mu \mathrm{m}$ ) on a 3axle micro milling unit. To realise a rule infringement the edges of the web are not rounded.

\subsection{2}

\section{Adapted UG menu bar}

To achieve a complete implementation of the system into the CAD system for a knowledge-based checking its menu bar has been correspondingly adapted. As a result, it now provides 5 new functionalities:

1. Selection of production technologies and type of machine element (Fig. 5)

2. Checking of the micro part and the dialogue box containing the result

3. Determination of the geometric properties of the current model

4. Access to the rule database

5. Access to the production database

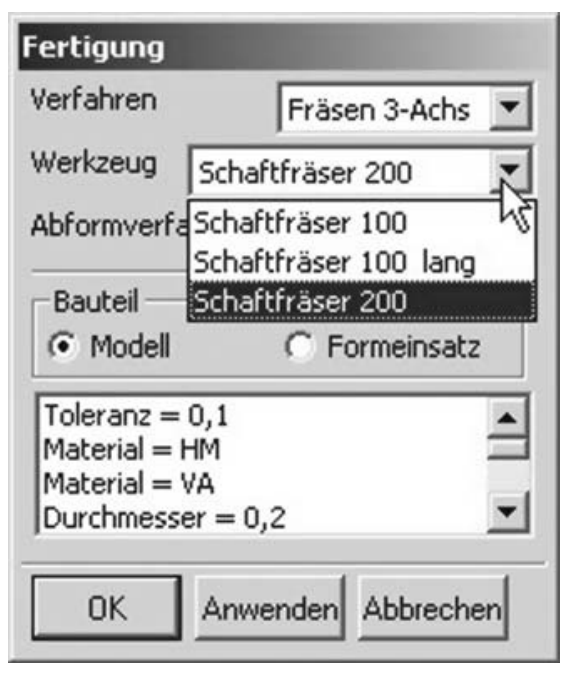

Fig. 5. Selection window for production technology and type of machine element 

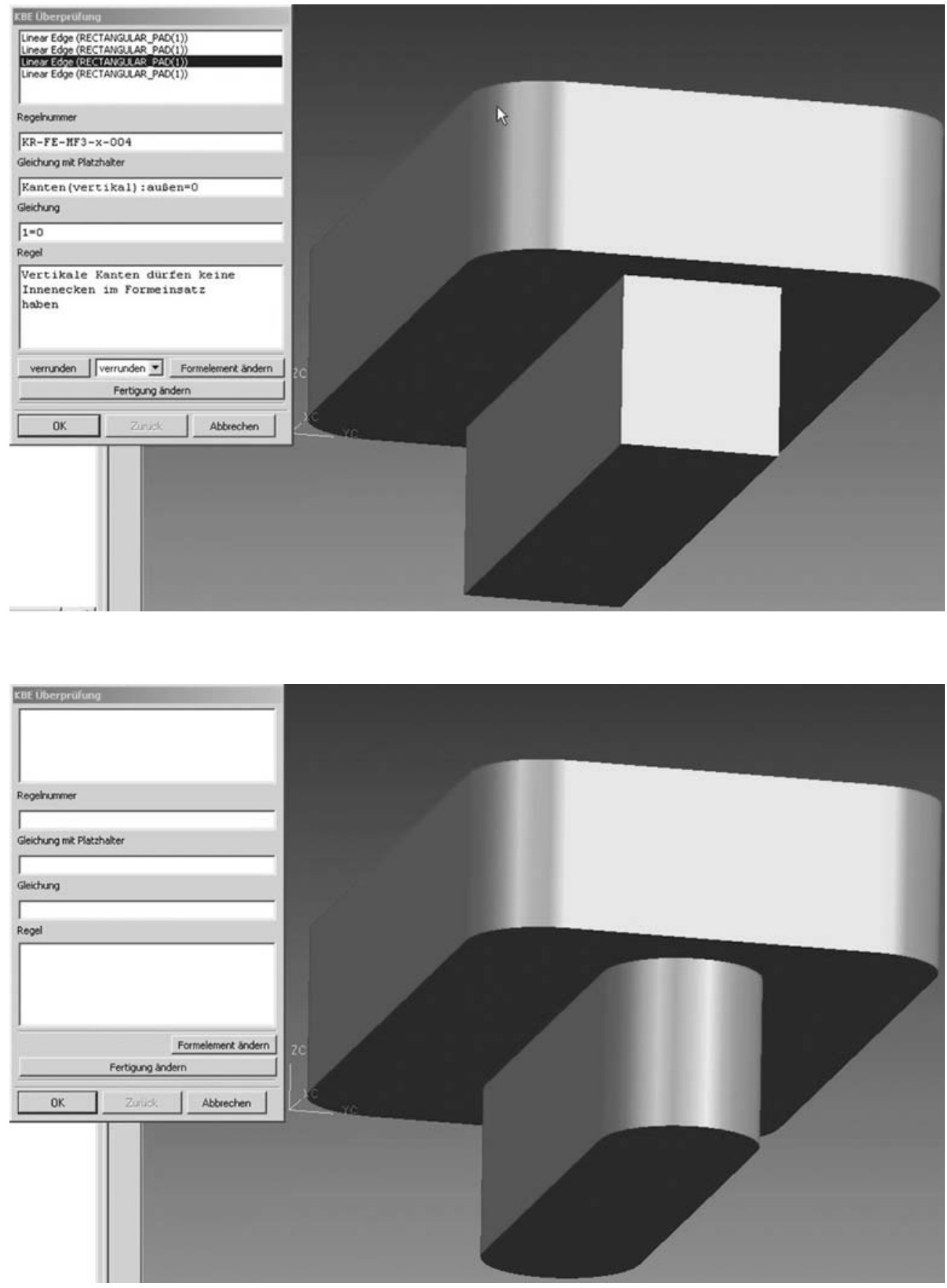

Fig. 6. Result window with infringed rules presented in a dialogue box
Fig. 7. After automatic correction (edge rounding) flawless micro part

\subsection{3}

\section{Checking the micro parts}

After the adjusting of the production technology and the type of machine element (Fig. 5) the checking is started and the result is later presented in a dialogue box (Fig. 6).

When an infringed rule is selected the matching geometric element is marked in the CAD model and additional information concerning the rule is displayed. In this case (not rounded edge) also an automatic correction is possible. If this is carried out for all four edges, the programme does not display an error message anymore

(Fig. 7). The model and the corresponding mold insert can be manufactured if the modifications are acceptable for the designer.
7

\section{Summary}

Unlike in mechanical engineering for the development of micro parts much more restrictions, above all those in production technology, need to be considered. [5] Therefore, providing a system for checking the geometry of the micro parts concerning certain parameter as a knowledgebased support for the designer is useful and necessary. Since this system is based on the knowledge about manufacturing quantities, material parameter and design rules, about which up to now information is only partly available, the system also requires an open structure and the possibility to easily modify the data record. For this purpose, a programme module has been implemented into the 
CAD system Unigraphics V18 [9], which is able to carry out this checking. The entire data record has been filed externally in databases. Thus, it is accessible for the user as well as for other systems independently of the CAD system.

The geometry analysis is based on the so-called "boundary representation" of the part [2], i.e. on the surfaces and edges (the boundary representations) of a solid. Hence, it is independent of the way how this solid has been generated and generally applicable for all CAD formats supporting this presentation (e.g. Parasolid, STEP) [3]. Although the analyse functions have been especially programmed for the demonstration objects they are universally applicable. Consequently, they also provide correct results for more complex parts, but do not nearly demonstrate all interesting geometric quantities. For this purpose, the set of functions needs to be extended.

The possibility to automatically carry out corrections in order to secure the production-compatible design of the micro part makes work easier for the designer. He is therefore able to concentrate on a function-oriented design as the system might automatically recognise and correct small deviations (e.g. roundings) which are less important for the function of the mechanical part but make production possible or clearly easier. As a result, unnecessary delays in the preparations or even deviations between the manufactured part and the drawing can be averted.

\section{References}

1. Albers A; Burkardt N; Marz J (2003) Restrictions in the design of gear wheel components and drives for micro technology.

Microsys Technol 9: 192-196 DOI 10.1007/s00542-002-0213-7
2. Cherian RP; Midha PS; Smith LN; Pipe AG (2001) Knowledge based and adaptive computational techniques for concurrent design of powder metallurgy parts. Advan Eng Soft 32: 455-465

3. DIN V EN V ISO 10303-42 (1994) Produktdatendarstellung und -austausch. Teil 42 Allgemeine integrierte Ressourcen: Geometrische und topologische Darstellung

4. Marz J (2001) Beschränkte Mikrowelt - Restriktionen bei der Konstruktion von Zahnradgetrieben für die Mikrotechnik. f\&m Feinwerktechnik Mikrotechnik Mikroelektronik 109: 51-52

5. Marz J; Burkardt N; Albers A (2003) Methodological investigation of the product development in micro technology. Proceedings of the 4th International Topical Conference, European Society for the Precision Engineering and Nanotechnologies (euspen): 23-26, May 19th/20th, Aachen, Germany

6. Ritzhaupt-Kleissl H-J; Bauer W; Knitter R; Piotter V; Ruprecht R; Haußelt J (2000) Fertigung keramischer Mikrokomponenten. 4. Statuskolloquium des Programms Mikrosytemtechnik, 30-31. März 2000, Wissenschaftliche Berichte FZKA 6423: 4550, Forschungszentrum Karlsruhe

7. Ruprecht R; Gietzelt T; Guttmann M; Hanemann T; Heckele M; Müller K; Piotter V (2000) Abformverfahren für mikrostrukturierte Bauteile aus Kunststoff und Metall. 4. Statuskolloquium des Programms Mikrosytemtechnik, 30-31. März 2000, Wissenschaftliche Berichte FZKA 6423: 39-44, Forschungszentrum Karlsruhe

8. Schmidt J; Simon M; Tritschler H; Ebner R (2001) $\mu$-Fräsen und $\mu$-Erodieren für den Formenbau. wt Werkstattstechnik online 91: 743-746

9. Unigraphics $\mathrm{CAD} / \mathrm{CAM} / \mathrm{CAE}-S y s t e m$ für die durchgängige virtuelle Produktentwicklung (VPD) (2002) http://www.ugsolutions.de/produkte/unigraphics.shtml 